\title{
Orthodontic Bond Strength Comparison between Two Filled Resin Sealants
}

\author{
James Anthony Kolstad' (D), Danielle Lynn Cianciolo ${ }^{2}$ (D), Alan James Ostertag ${ }^{3}$ (D), David William Berzins ${ }^{4}$ (1)
}

'Orthodontic Resident, Department of Developmental Sciences, Marquette University School of Dentistry, Milwaukee, Wisconsin, USA

2Marquette University School of Dentistry, Milwaukee, Wisconsin, USA

${ }^{3}$ Private Practice, Eau Claire, Wisconsin, USA

${ }^{4}$ General Dental Sciences, Marquette University School of Dentistry, Milwaukee, Wisconsin, USA

Cite this article as: Kolstad JA, Cianciolo DL, Ostertag AJ, Berzins DW. Orthodontic Bond Strength Comparison between Two Filled Resin Sealants. Turk J Orthod 2020; 33(3): 165-70.

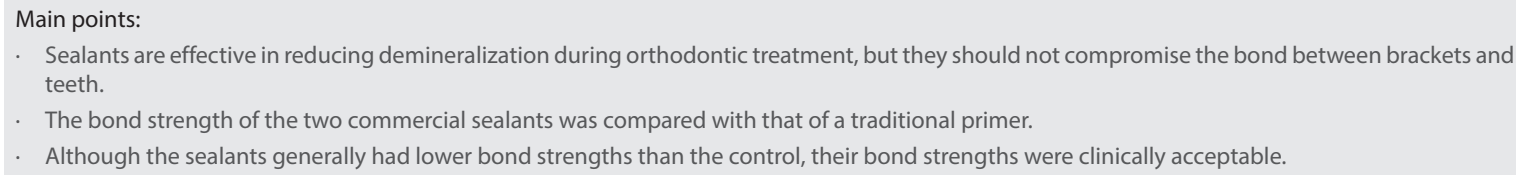

\section{ABSTRACT}

Objective: Sealants are used in orthodontics to help prevent demineralization during treatment. This study aimed to determine if there is a difference in the shear bond strength (SBS) between 2 different resin sealants bonded to teeth.

Methods: Extracted human premolars ( $n=20 /$ group) were randomly divided and prepared by acid etching, followed by application of primer or sealant. Group 1, the control group, used Transbond XT Primer (3M Unitek). Groups 2 and 3 were prepared with the sealants L.E.D. Pro Seal (Reliance Orthodontic Products) and Opal Seal (Opal Orthodontics) as the respective primers. Transbond XT Adhesive was applied to a stainless steel bracket and bonded to each tooth. Each group was stored in distilled water at $37^{\circ} \mathrm{C}$ for 48 hours before. SBS was measured using a universal testing machine, and the adhesive remnant index (ARI) was scored.

Results: The SBS (MPa) of the groups was as follows: Group 1 (Transbond): $20.1 \pm 6.0$; Group 2 (Pro Seal): $16.5 \pm 4.8$; and Group 3 (Opal Seal): $15.7 \pm 3.9$. The SBS of Transbond XT Primer was significantly greater than that of Opal Seal ( $p<0.05 / a n a l y s i s$ of variance-Tukey), while Pro Seal and Opal Seal sealants were not significantly different from each other $(p<0.05)$. The Opal Seal group had significantly greater ARI scores, indicating that more adhesive remained on the teeth after debonding.

Conclusion: Opal Seal and Pro Seal sealants have similar SBS but generally exhibit lower bond strengths than an adhesive primer.

Keywords: Adhesive remnant index, bond strength, orthodontic sealant

\section{INTRODUCTION}

A common problem in orthodontic treatment is the formation of white spot lesions or enamel decalcification on the tooth. The prevalence of white spot lesions in orthodontic patients has been shown to be 34\%-97\%, whereas the incidence of such lesions during orthodontic therapy has been shown to be $23 \%-76 \%$ (1). White spot lesions are considered to be unhealthy, irreversible, and unesthetic (2-4). Patients, parents, orthodontists, and dentists agree that white spot lesions detract from the overall appearance of the orthodontic patient, and the patient is primarily responsible for the prevention of these lesions (4). Nevertheless, white spot lesions are easily detectable and can be arrested by preventive treatment or even prevented altogether (5).

Over the years, orthodontists have tried many different ways to prevent enamel demineralization in their patients $(2,3,5-11)$. Prevention methods have included oral hygiene instruction, fluoride mouth rinses, application 
of fluoride varnishes, and sealants. All the preventive methods, other than fluoride varnish and sealants, require patient compliance during treatment (2). It has been shown that a relationship may exist between patient compliance and the formation of white spot lesions $(4,5,11)$. One way to combat the need for patient compliance and reduce decalcification is the application of a sealant on the facial aspect of the tooth before bonding the bracket $(3,9)$. Opal Seal (Opal Orthodontics, South Jordan, UT, USA) and L.E.D. Pro Seal (Reliance Orthodontic Products, Itasca, IL, USA) are two different brands of orthodontic sealants.

Pro Seal is described by the manufacturer as a fluoride-containing, light-cured filled sealant that completely sets without an oxygen-inhibited layer, creating a smooth and hard surface that prevents leakage and protects the enamel (12). Opal Seal is $38 \%$ filled with proprietary glass ionomer fillers and nanofillers and is also light-curable and contains fluoride (13). Both the sealants contain a fluorescing agent that can be illuminated by a black light to determine whether the sealant is still present on the tooth surface $(12,13)$. Recent independent in vitro studies have evaluated Pro Seal and Opal Seal sealants for their surface, mechanical, and esthetic properties $(3,7,10)$. Results from these studies have shown that each sealant may have advantages over the other. Opal Seal was found to be significantly harder, allowed less Streptococcus mutans adherence, and had better color stability $(7,10)$. In contrast, Pro Seal was found to be more wear-resistant and released significantly greater amounts of fluoride ( 7 , 10). In terms of efficacy, both Pro Seal and Opal Seal sealants provide reductions in enamel demineralization compared with the untreated controls $(3,6,14)$.

Understanding the different properties of each product along with their bond strength can play an important role in deciding which product to use clinically. Although some of the physical and esthetic properties of each sealant have been compared with each other, their orthodontic bond strengths have not been compared. Research has been conducted to investigate the bond strength of Pro Seal sealant bonded with different adhesives $(5,9,15-18)$. For example, Lowder et al. (9) found that Pro Seal sealant produced clinically acceptable bond strengths when coupled with four different adhesives, but its bond strength was lower than two regular primer/adhesive systems. Comparatively, the bond strength of Opal Seal sealant has not been investigated as thoroughly (19). This study aimed to compare the shear bond strength (SBS) between two different resin sealants when used to bond orthodontic brackets to teeth. The null hypothesis was that there would be no difference in SBS between Pro Seal and Opal Seal.

\section{METHODS}

Following the Institutional Review Board clearance (Approval No: DT-027), 60 human premolar teeth extracted for orthodontic reasons were collected and stored in distilled water at $4^{\circ} \mathrm{C}$. Each patient or parent for a minor patient signed a consent form allowing for their teeth to be used for research purposes. The extracted teeth possessed no identifying information; therefore, the age of the patient was not known to the researchers. How- ever, the teeth consisted of upper and lower, first and second premolars. The distilled water was refreshed periodically to limit bacterial growth, and the time required to collect all necessary teeth was 6 months. If any large restorations, enamel defects, or any abnormal flaws were found on examination, the tooth was excluded. The roots were removed from each tooth with a highspeed handpiece and diamond bur. The cut was made about 6 $\mathrm{mm}$ below the cementoenamel junction. Each crown was then placed back into a container of distilled water at $4^{\circ} \mathrm{C}$.

The teeth were randomly divided into 3 groups ( $n=20 /$ group). Randomization was achieved by mixing the 60 extracted premolars and blindly selecting the teeth to comprise each group in a parallel manner (tooth 1 for each group sequentially to tooth 20 for each group). Group 1 was bonded with Transbond XT Primer (3M Unitek, Monrovia, CA, USA) and Transbond XT light cure adhesive (3M Unitek). Group 2 was bonded with L.E.D. Pro Seal sealant and Transbond XT adhesive. Group 3 was bonded with Opal Seal sealant and Transbond XT Adhesive. Stainless steel brackets (universal upper bicuspid, Victory Series, 3M Unitek) with zero torque and tip were used. The surface area of the bracket base was $10 \mathrm{~mm}^{2}$.

Before the bonding procedure, each tooth was cleaned with a rubber prophy cup on a slow-speed handpiece with pumice paste (Nada, Preventive Technologies, Inc., Indian Trail, NC, USA) for 5 seconds and then rinsed with water. The tooth was then etched using 35\% phosphoric acid etching gel (3M Unitek) for 30 seconds and was thoroughly rinsed and dried until the etched buccal surface appeared frosty white. For each group, the primer or sealant (Transbond XT Primer, Pro Seal, and Opal Seal for Groups 1-3, respectively) was applied to the buccal surface of the tooth following manufacturer instructions. Transbond XT Adhesive was then applied to the bracket base. The bracket was placed in the proper position on the tooth and was pressed firmly to seat the bracket. The excessive resin was removed, and the adhesive was light-cured (Ortholux Luminous Curing Light, 3M Unitek) for 10 seconds on both the mesial and distal aspects of the bracket. One operator prepared all the teeth. The tooth with the bonded bracket was then placed back into the appropriate container of distilled water and stored at $37^{\circ} \mathrm{C}$ for 24 hours.

After storage, the teeth were individually mounted in cold-cure acrylic (Great Lakes Orthodontics, Tonawanda, NY, USA). Each tooth was attached to a 0.018 -inch stainless steel wire using an elastomeric module and suspended over a small section of polyvinyl chloride pipe. The acrylic was mixed and poured into the pipe to the level of the cusp tip of the suspended tooth, assuring each tooth was mounted in the acrylic in a repeatable way. After the acrylic set, each bonded and mounted tooth was placed back into distilled water and stored at $37^{\circ} \mathrm{C}$ for 24 hours.

A universal testing machine (Instron, Norwood, MA, USA) was used to measure the SBS of each bracket/tooth specimen. Each mounted tooth was secured in a fixture that allowed a blade attached to the machine crosshead to contact the bracket between its base and gingival tie wings (Figure 1). A shear force 
Table 1. Shear bond strength and Weibull analysis

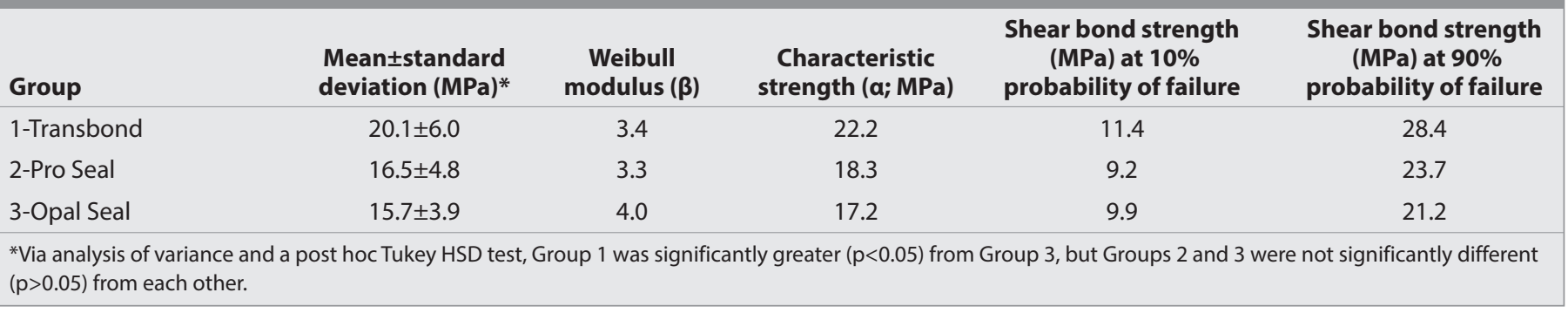
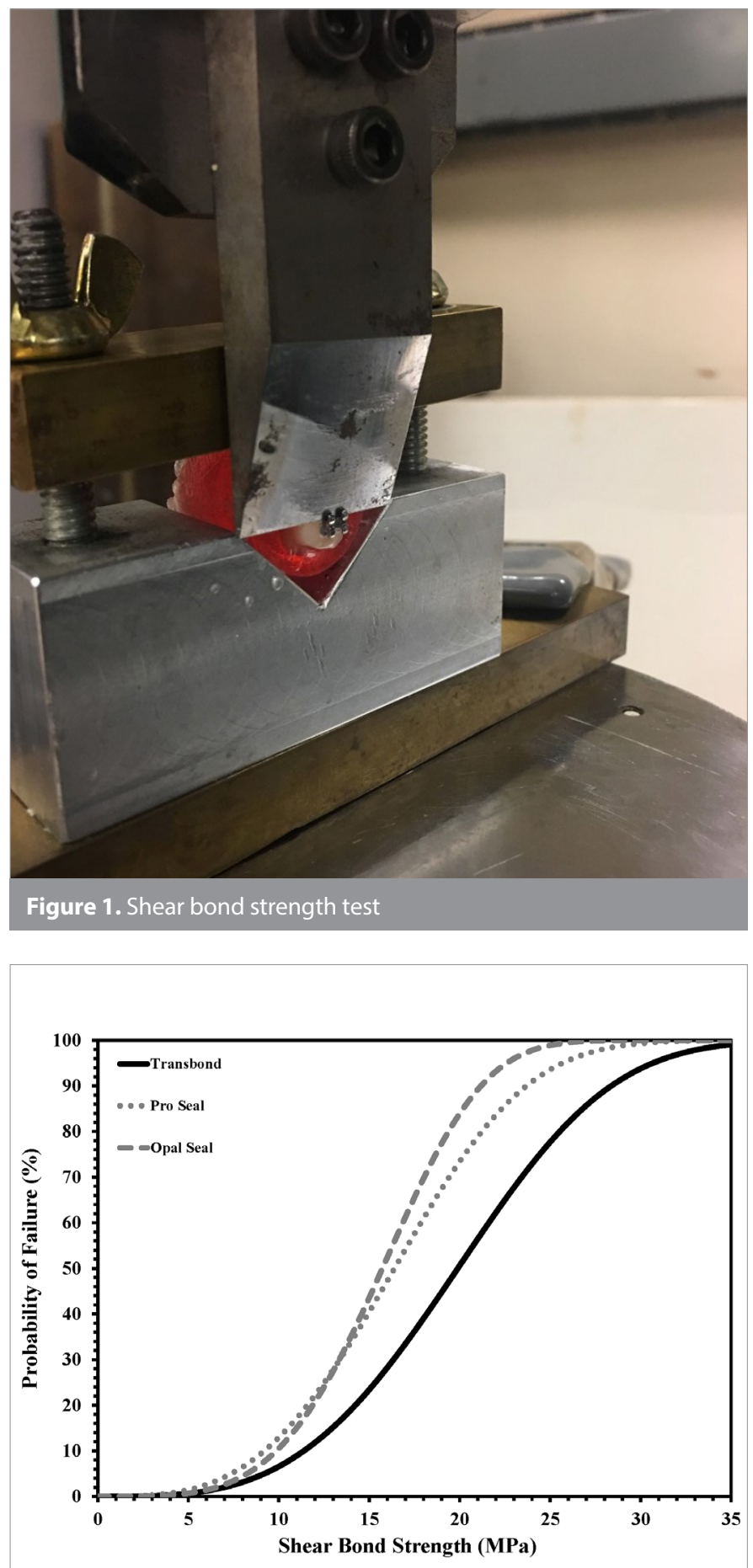

Figure 2. Weibull curves for the shear bond strength of the three groups
Table 2. Comparison of Adhesive remnant index (ARI) scores among groups by Kruskal-Wallis and Mann-Whitney U tests.

\begin{tabular}{lcccc|} 
& \multicolumn{4}{c|}{ ARI scores* } \\
\cline { 2 - 5 } Group & $\mathbf{0}$ & $\mathbf{1}$ & $\mathbf{2}$ & $\mathbf{3}$ \\
1-Transbond & 0 & 10 & 10 & 0 \\
2-Pro Seal & 0 & 9 & 11 & 0 \\
3-Opal Seal & 0 & 0 & 20 & 0 \\
*There was no significant difference $(\mathrm{p}>0.05)$ between Groups 1 and 2; how- \\
ever, Group 3 was significantly different $(\mathrm{p}=0.001)$
\end{tabular}

at a crosshead speed of $0.5 \mathrm{~mm} / \mathrm{min}$ was used to debond each bracket. The force was measured in Newtons and converted to MPa by dividing by the bracket base area.

After each bracket was debonded, the enamel surface and bracket were examined using an optical microscope and scored using the adhesive remnant index (ARI) (20). The ARI score represents the amount of adhesive remaining on the enamel after debonding the bracket. There are 4 possible ARI scores: $0=$ no adhesive left on the tooth, $1=$ less than $50 \%$ of the adhesive left on the tooth, $2=$ more than $50 \%$ of the adhesive left on the tooth, and $3=$ all of the adhesive left on the tooth.

SBS was analyzed using one-way analysis of variance and a post hoc Tukey HSD test at $p \leq 0.05$ level of significance. ARI data were compared using Kruskal-Wallis and Mann-Whitney $U$ tests via the Statistical Package for Social Sciences version 23.0 software (IBM Corp.; Armonk, NY, USA).

\section{RESULTS}

The SBSs (MPa) of the groups are listed in Table 1. The SBS for Transbond XT Primer, was significantly greater than that for Opal Seal sealant $(p<0.05)$, but Pro Seal and Opal Seal sealants were not significantly different from each other $(p<0.05)$. Weibull analysis also indicated that the Transbond XT Primer group displayed greater bond strengths. However, Opal Seal possessed the greatest Weibull modulus, indicating slightly greater reliability between the groups as it had less broadly distributed bond strength values. This is further reflected in the lower standard deviation for the Opal Seal group. Figure 2 displays Weibull curves plotting "Probability of Failure" versus Shear Bond Strength that are consistent with Table 1. In terms of bond failure site, the Opal Seal group had significantly greater ARI scores ( $p=0.001$; Table 2$)$, indicating that more adhesive remained on the tooth after bond strength testing. 


\section{DISCUSSION}

The purpose of this study was to determine if there was a difference in SBS between Pro Seal and Opal Seal sealants. Previous studies have shown that Pro Seal sealant exhibited clinically acceptable bond strength and compared different properties of Pro Seal and Opal Seal sealants $(3,7,9,10)$. The literature shows that there is an added benefit to using a sealant in the protection against the formation of white spot lesions. Specifically, Tasios et al. (21) conducted a meta-analysis to assess the efficacy of preventive interventions against the development of white spot lesions and found that sealants, active patient reminders, and fluoride varnishes were associated with reduced white spot lesion incidence. Five randomized clinical trials were included in their analysis that supported the use of sealants. However, there has not been a study that has compared the bond strength of Opal Seal sealant to Pro Seal sealant.

Results showed that the two orthodontic sealants performed similarly with respect to SBS; thus, the null hypothesis was accepted, although the adhesive primer (control) group had a statistically greater SBS than Opal Seal sealant group. Transbond XT Adhesive with Transbond XT Primer has been regarded as the gold standard when bonding to enamel (22). Nevertheless, both Pro Seal and Opal Seal sealants had SBS over $15 \mathrm{MPa}$, which is considered clinically acceptable according to Tavas and Watts who stated that bond strength of $6 \mathrm{kgf}$ was needed in 24 hours (23). For comparison, the average SBS of Opal Seal at 15.7 MPa corresponds to $16 \mathrm{kgf}\left(15.7 \mathrm{MPa} \times 10 \mathrm{~mm}^{2} / 9.8 \mathrm{~m} / \mathrm{s}^{2}\right)$. Comparatively, the force levels for debonding the brackets in this study using Transbond XT Primer and Pro Seal sealant were slightly higher than those reported by Lowder et al. (9). In the study by Lowder et al. (9), the specimens were stored for 30 days and thermocycled, both of which are factors that have been shown to decrease the bond strength (24-26). Furthermore, the crosshead speed was slower in this study, although the effect of crosshead speed on orthodontic bond strength has been inconsistent (27-29).

ARI is one of the most commonly used methods to determine the quality of adhesion at the bracket/adhesive and tooth/adhesive interfaces. The ARI results for the Transbond XT Primer and Pro Seal sealant groups were quite evenly split between ARI 1 and 2 scores, whereas Opal Seal sealant had a significantly greater ARI score, indicating that more amount of adhesive consistently remained on the teeth after debonding. Although the exact composition and concentration of all monomers in the products are proprietary, the Safety Data Sheets list Opal Seal sealant and Transbond XT Adhesive as containing bisphenol A-glycidyl methacrylate, whereas Pro Seal sealant does not contain the same product. Opal Seal sealant and Transbond XT Adhesive may have better compatibility, thereby forming a stronger bond and shifting the weak link onto the bracket/adhesive interface than the other two groups. However, more research is needed to confirm this. While more adhesive left on the tooth may lower the risk of enamel fracture, it would also increase the clean-up time by the orthodontist. This study used standard stainless steel brackets that required application of adhesive to the bracket base; use of a different bracket system may alter the adhesive failure site. For instance, a recent study found that precoated brackets had lower ARI scores than the conventional brackets (30). This can be attributed to the fact that precoated brackets have a premeasured uniform layer of adhesive. Alternatively, the lower ARI scores may also be the result of the more uniform pressure that is applied in placing the adhesive on the bracket mesh during manufacturing, allowing for better penetration of the mesh (30). Failures at the bracket/adhesive interface may also be caused by the incomplete polymerization of the adhesive owing to lack of light curing behind the bracket.

Orthodontic literature outlines different factors that influence bond strength and ARI (24). Those factors include operator technique, patient behavior, enamel variations, specimen storage time, enamel conditioning procedures, type of adhesive, and bracket base area/design (24). In this study, all the materials and processes were the same except for the primer/sealants being compared. Protocols from the study by Fox et al. (31) were used to help with standardization of this study. As this was an in vitro study, there were limitations to translating the current research to clinical practice. Thermocycling is frequently performed in orthodontic bonding studies to serve as an artificial aging mechanism to gain insight on long-term bond strength. Thermocycling has been found to decrease the orthodontic bond strength in a majority of studies, but it is not always observed $(26,32-35$, 36-38). Nevertheless, the SBS values reported in this study were at 48 hours after bonding without thermocycling; thus, the results do not represent longer conditions that may be of greater interest. Furthermore, the upper and lower premolars were used in this study without stratification. Generalization to other teeth is problematic because enamel shape and tooth type influence the bond strength $(39,40-42)$; however, two of these studies found no difference in bond strength between upper and lower premolars $(40,41)$ in contrast to the study by Ozturk et al. $(42)$. Therefore, a clinical comparison of the two sealants is necessary to properly ascertain their demineralization efficacy and bonding durability.

\section{CONCLUSION}

Opal Seal and Pro Seal sealants have similar SBS but generally exhibit lower bond strengths than adhesive primer. Opal Seal sealant leaves more adhesive on the tooth when debonding occurs, which could lead to an increase in debond appointment time.

Ethics Committee Approval: This study was approved by the Institutional Review Board of Marquette University (Approval No: DT-027).

Informed Consent: Written informed consent was obtained from the patients who agreed to donate their extracted teeth for research purposes.

Peer-review: Externally peer-reviewed.

Author Contributions: Supervision - A.O., D.B.; Design - J.K., A.O., D.B.; Resources - J.K., D.B.; Materials - J.K., D.B.; Data Collection and/or Processing - J.K., D.C., D.B.; Analysis and/or Interpretation - J.K. D.B.; Literature Search - J.K., D.B.; Writing Manuscript - J.K., D.B.; Critical Review - D.C., A.O. 
Conflict of Interest: The authors have no conflict of interest to declare.

Financial Disclosure: The authors declared that this study has received no financial support.

\section{REFERENCES}

1. Sundararaj D, Venkatachalapathy S, Tandon A, Pereira A. Critical evaluation of incidence and prevalence of white spot lesions during fixed orthodontic appliance treatment: A meta-analysis. J Int Soc Prev Community Dent 2015; 5: 433-9. [Crossref]

2. Oz AZ, Oz AA, Yazıcıoglu S. In vivo effect of antibacterial and fluoride-releasing adhesives on enamel demineralization around brackets: A micro-CT study. Angle Orthod 2017; 87: 841-6. [Crossref]

3. Premaraj TS, Rohani N, Covey D, Premaraj S, Hua Y, Watanabe H. An in-vitro evaluation of mechanical and esthetic properties of orthodontic sealants. Eur J Dent 2014; 8: 487-92. [Crossref]

4. Behnan SM, Arruda AO, González-Cabezas C, Sohn W, Peters MC. In-vitro evaluation of various treatments to prevent demineralization next to orthodontic brackets. Am J Orthod Dentofacial Orthop 2010; 138: 712.e1-7; discussion 712-3. [Crossref]

5. Hu W, Featherstone JD. Prevention of enamel demineralization: An in-vitro study using light-cured filled sealant. Am J Orthod Dentofacial Orthop 2005; 128: 592-600. [Crossref]

6. Buren JL, Staley RN, Wefel J, Qian F. Inhibition of enamel demineralization by an enamel sealant, Pro Seal: An in-vitro study. Am J Orthod Dentofacial Orthop 2008; 133(4 Suppl): 88-94. [Crossref]

7. Maxfield BJ, Hamdan AM, Tüfekçi E, Shroff B, Best AM, Lindauer SJ. Development of white spot lesions during orthodontic treatment: perceptions of patients, parents, orthodontists, and general dentists. Am J Orthod Dentofacial Orthop 2012; 141: 337-44. [Crossref]

8. Bishara SE, Oonsombat C, Soliman MM, Warren J. Effects of using a new protective sealant on the bond strength of orthodontic brackets. Angle Orthod 2005; 75: 243-6.

9. Lowder PD, Foley T, Banting DW. Bond strength of 4 orthodontic adhesives used with a caries-protective resin sealant. Am J Orthod Dentofacial Orthop 2008; 134: 291-5. [Crossref]

10. Premaraj TS, Rohani N, Covey D, Premaraj S. In vitro evaluation of surface properties of Pro Seal $^{\circledR}$ and Opal ${ }^{\circledR}$ SealTM in preventing white spot lesions. Orthod Craniofac Res 2017; 20: 134-8. [Crossref]

11. Hadler-Olsen S, Sandvik K, El-Agroudi MA, Øgaard B. The incidence of caries and white spot lesions in orthodontically treated adolescents with a comprehensive caries prophylactic regimen--a prospective study. Eur J Orthod 2012; 34: 633-9. [Crossref]

12. Product Catalog. Reliance Orthodontic Products, Inc. 2020.

13. Opal Seal Primer and Sealer IFU. Ultradent Products, Inc. 2017.

14. Bartels AA, Evans CA, Viana G, Bedran-Russo AK. Demineralization of resin-sealed enamel by soft drinks in a clinically relevant $\mathrm{pH}$ cycling model. Am J Dent 2016; 29: 115-9.

15. Tarvade SM, Deshmukh AA, Daokar SG. Evaluation of Bond Strength: An In vitro Study using Pro Seal. J Int Oral Health 2014; 6: 1-3.

16. Paschos E, Okuka S, Ilie N, Huth KC, Kickel R, Rudzki-Janson I. Investigation of shear-peel bond strength of orthodontic brackets on enamel after using Pro Seal. J Orofac Orthop 2006; 67: 196-206. [Crossref]

17. El Bokle D, Munir H. An in vitro study of the effect of Pro Seal varnish on the shear bond strength of orthodontic brackets. World J Orthod 2008; 9: 141-6.

18. Phan X, Akyalcin S, Wiltshire WA, Rody WJ Jr. Effect of tooth bleaching on shear bond strength of a fluoride-releasing sealant. Angle Orthod 2012; 82: 546-51. [Crossref]
19. Seeliger JH, Botzenhart UU, Gedrange T, Kozak K, Stepien L, Machoy $M$. Enamel shear bond strength of different primers combined with an orthodontic adhesive paste. Biomed Tech (Berl) 2017; 62: 415-20. [Crossref]

20. Artun J, Bergland S. Clinical trials with crystal growth conditioning as an alternative to acid-etch enamel pretreatment. Am J Orthod 1984; 85: 333-40. [Crossref]

21. Tasios T, Papageorgiou SN, Papadopoulos MA, Tsapas A, Haidich AB. Prevention of orthodontic enamel demineralization: A systematic review with meta-analyses. Orthod Craniofac Res 2019; 22: 225-35. [Crossref]

22. Hellak A, Ebeling J, Schauseil M, Stein S, Roggendorf M, Korbmacher-Steiner $\mathrm{H}$. Shear bond strength of three orthodontic bonding systems on enamel and restorative materials. Biomed Res Int 2016; 2016: 6307107. [Crossref]

23. Tavas MA, Watts DC. A visible light-activated direct bonding material: An in vitro comparative study. $\mathrm{Br} J$ Orthod 1984; 11: 33-7. [Crossref]

24. Murray SD, Hobson RS. Comparison of in vivo and in vitro shear bond strength. Am J Orthod Dentofacial Orthop 2003; 123: 2-9. [Crossref]

25. Oesterle LJ, Shellhart WC. Effect of aging on the shear bond strength of orthodontic brackets. Am J Orthod Dentofacial Orthop 2008; 133: 716-20. [Crossref]

26. Sokucu O, Siso SH, Ozturk F, Nalcaci R. Shear bond strength of orthodontic brackets cured with different light sources under thermocycling. Eur J Dent 2010; 4: 257-62. [Crossref]

27. Bishara SE, Soliman M, Laffoon J, Warren JJ. Effect of changing a test parameter on the shear bond strength of orthodontic brackets. Angle Orthod 2005; 75: 832-5.

28. Klocke A, Kahl-Nieke B. Influence of cross-head speed in orthodontic bond strength testing. Dent Mater 2005; 21: 139-44. [Crossref]

29. Shooter KJ, Griffin MP, Kerr B. The effect of changing crosshead speed on the shear bond strength of orthodontic bonding adhesive. Aust Orthod J 2012; 28: 44-50.

30. Guzman UA, Jerrold L, Vig PS, Abdelkarim A. Comparison of shear bond strength and adhesive remnant index between precoated and conventionally bonded orthodontic brackets. Prog Orthod 2013; 14: 39. [Crossref]

31. Fox NA, McCabe JF, Buckley JG. A critique of bond strength testing in orthodontics. Br J Orthod 1994; 21: 33-43. [Crossref]

32. Arici S, Arici N. Effects of thermocycling on the bond strength of a resin-modified glass ionomer cement: an in vitro comparative study. Angle Orthod 2003; 73: 692-6.

33. Bishara SE, Ajlouni R, Laffoon JF. Effect of thermocycling on the shear bond strength of a cyanoacrylate orthodontic adhesive. Am J Orthod Dentofacial Orthop 2003; 123: 21-4. [Crossref]

34. Lopes GV, Correr-Sobrinho L, Correr AB, Godoi APT, Vedovello SAS, Menezes CC. Light activation and thermocycling methods on the shear bond strength of brackets bonded to porcelain surfaces. Braz Dent J 2020; 31: 52-6. [Crossref]

35. Turk T, Elekdag-Turk S, Isci D, Cakmak F, Ozkalayci N. Shear bond strength of a self-etching primer after 10,000 and 20,000 thermal cycles. J Adhes Dent 2010; 12: 117-22.

36. Costa AR, Correr AB, Puppin-Rontani RM, Vedovello SA, Valdrighi $H C$, Correr-Sobrinho $L$, et al. Effects of thermocycling and light source on the bond strength of metallic brackets to bovine teeth. Braz Dent J 2011; 22: 486-9. [Crossref]

37. Yuasa T, lijima M, Ito $S$, Muguruma T, Saito T, Mizoguchi I. Effects of long-term storage and thermocycling on bond strength of two self-etching primer adhesive systems. Eur J Orthod 2010; 32: 28590. [Crossref] 
38. Cerekja E, Cakirer B. Effect of short curing times with a high-intensity light-emitting diode or high-power halogen on shear bond strength of metal brackets before and after thermocycling. Angle Orthod 2011;81: 510-6. [Crossref]

39. Hioki M, Shin-Ya A, Nakahara R, Vallittu PK, Nakasone Y, Shin-Ya A. Shear bond strength and FEM of a resin-modified glass ionomer cement--effects of tooth enamel shape and orthodontic bracket base configuration. Dent Mater J 2007; 26: 700-7. [Crossref]
40. Hobson RS, McCabe JF, Hogg SD. Bond strength to surface enamel for different tooth types. Dent Mater 2001; 17: 184-9. [Crossref]

41. Linklater RA, Gordon PH. An ex vivo study to investigate bond strengths of different tooth types. J Orthod 2001; 28: 59-65. [Crossref]

42. Oztürk B, Malkoç S, Koyutürk AE, Catalbas B, Ozer F. Influence of different tooth types on the bond strength of two orthodontic adhesive systems. Eur J Orthod 2008; 30: 407-12. [Crossref] 